\title{
Steady-state entanglement of two atoms created by classical driving field
}

\author{
Özgür Çakir, Alexander A. Klyachko, and Alexander S. Shumovsky \\ Faculty of Science, Bilkent University, Bilkent, Ankara 06800, Turkey
}

(Received 12 June 2004; published 10 March 2005)

\begin{abstract}
The stabilization of entanglement caused by action of a classical driving field in the system of two-level atoms with the dipole interaction accompanied by spontaneous emission is discussed. An exact solution shows that the maximum amount of concurrence that can be achieved in the Lamb-Dicke limit is 0.43 . Dependence of entanglement on interatomic distance and the classical driving field, beyond the Lamb-Dicke limit, is examined numerically.
\end{abstract}

DOI: 10.1103/PhysRevA.71.034303

PACS number(s): 03.67.Mn, 03.65.Ud, 32.80.-t, 42.50.Ct

The main aim of this paper is to show that classical driving field can be used to stabilize entanglement in atomic systems.

It is well known that two-level atoms can be successfully used to model entangled states of qubits as well as realization of different quantum communication protocols. In fact, two-level atoms have been used for decades as the main tool for testing fundamentals of quantum mechanics (see Refs. $[1,2]$ and references therein).

The practical applications require the robust entangled states. This notion includes long enough lifetime of the states and high amount of entanglement (as close to perfect entanglement as possible). However, in many cases entanglement of two-level atoms is not stable enough. In the case of atoms trapped in high-quality cavities, absence of stability is caused mainly by Rabi oscillations. In free space, entanglement related to exited atomic states decays because of the spontaneous emission processes.

To stabilize atomic entanglement, an engineered environment can be utilized. For example, it was shown in Refs. [3-5] that the presence of a squeezed vacuum field can stabilize entanglement of a pair of two-level atoms with dipoledipole interaction. The use of a bad cavity as a stabilizing environment was considered in Ref. [6]. Stabilization in a bad cavity with optical white-noise field was discussed in Ref. [7]. A scheme of stabilization based on the use of threelevel $\Lambda$-type atoms in two-mode cavities with leakage and absorption was proposed in Ref. [8] and then discussed in Ref. [9].

In this note, we show that a reasonable amount of steadystate entanglement can be achieved in a system of two-level atoms in the weak-coupling regime (high losses), in particular for free space, in the presence of a classical driving field. The collective effects, i.e., dipole-dipole interaction and collective spontaneous emission, are the mechanisms responsible for generation of entanglement. However, in the absence of a special environment that compensates the losses of energy caused by spontaneous emission, the entanglement is a transient one. We show that instead of a more sophisticated squeezed vacuum field the simple classical driving field can be succesfully used for this aim. The classical driving field alone acts only locally on the atoms, so that it cannot create specific quantum correlations between the atoms peculiar for the entangled state. However, it continuously provides atomic excitations that are responsible for survival of the collective effects thus enabling a steady-state entanglement.

The system of two identical two-level atoms in free space is governed by the master equation [10]

$$
\begin{aligned}
\dot{\rho}=- & i[H, \rho]+\frac{1}{2} \sum_{i, j=1}^{2} \Gamma_{i j}\left(2 \sigma_{-}^{i} \rho \sigma_{+}^{j}-\sigma_{+}^{i} \sigma_{-}^{j} \rho-\rho \sigma_{+}^{i} \sigma_{-}^{j}\right), \\
H= & \sum_{i=1}^{2}\left[\frac{\omega}{2} \sigma_{z}^{i}+E\left(\sigma_{+}^{i} e^{i \vec{k} \cdot \vec{r}_{i}-i \omega t}+\sigma_{-}^{i} e^{-i \vec{k} \cdot \vec{r}_{i}+i \omega t}\right)\right] \\
& +\Omega\left(\sigma_{+}^{1} \sigma_{-}^{2}+\sigma_{+}^{2} \sigma_{-}^{1}\right),
\end{aligned}
$$

where the atomic dipoles are aligned in the same direction along the interatomic axis and driven by a linearly polarized classical field, with dipole coupling constant $E$. Here $\sigma_{+}^{i}$ $=\left(\sigma_{-}^{i}\right)^{\dagger}=|e\rangle_{i}\left\langle\left. g\right|_{i} \text { and } \sigma_{z}^{i}=\mid e\right\rangle_{i}\left\langle\left. e\right|_{i}-\mid g\right\rangle_{i}\left\langle\left. g\right|_{i} \text { with } \mid e\right\rangle_{i},|g\rangle_{i}$ denoting the excited and the ground states of the $i$ th atom, $\Gamma_{i i}=\Gamma$ is the single atom decay rate, $\Gamma=\omega^{3}|\vec{\mu}|^{2} / 3 \pi \hbar \epsilon_{0} c^{3}$, and $\vec{\mu}$ is the atomic dipole moment. The collective decay rates are

$$
\Gamma_{12}=\Gamma_{21}=-3 \Gamma\left[\frac{\cos k r}{(k r)^{2}}-\frac{\sin k r}{(k r)^{3}}\right],
$$

and the coupling constant for dipole-dipole interaction has the form

$$
\Omega=-\frac{3}{2} \Gamma\left(\frac{\sin k r}{(k r)^{2}}+\frac{\cos k r}{(k r)^{3}}\right) .
$$

We are going to consider the case when the classical field is in phase at the atomic locations, namely $\vec{k} \cdot \vec{r}_{12}=0$. If the density matrix is initially block diagonal,

$$
\rho=\left[\begin{array}{cc}
\rho_{T} & 0 \\
0 & \rho_{S}
\end{array}\right] \rightarrow \rho_{T}=\left[\begin{array}{lll}
\rho_{11} & \rho_{12} & \rho_{13} \\
\rho_{21} & \rho_{22} & \rho_{23} \\
\rho_{31} & \rho_{32} & \rho_{33}
\end{array}\right], \quad \rho_{S}=\rho_{44},
$$

in the total angular momentum basis, consisting of the four states $\{|e e\rangle,|s\rangle=(|e g\rangle+|g e\rangle) / \sqrt{2},|a\rangle=(|e g\rangle-|g e\rangle) / \sqrt{2},|g g\rangle\}$, then it will always preserve the block diagonal form. Here $\rho_{T}$ is defined in the triplet part of the Hilbert space spanned by the symmetric vectors in the above basis, while $\rho_{S}$ corresponds to singlet subspace with antisymmetric base vector 
$|a\rangle$. This fact directly follows from the equations of motion for $\rho_{T}$ and $\rho_{S}$,

$$
\begin{aligned}
& \dot{\rho}_{T}=-i\left(H_{T} \rho_{T}-\rho_{T} H_{T}^{\dagger}\right)+\frac{\Gamma+\Gamma_{12}}{2} J_{-} \rho_{S} J_{+} \\
&+\left(\Gamma-\Gamma_{12}\right) \rho_{S}|g g\rangle\langle g g|, \\
& \dot{\rho}_{S}=-\left(\Gamma-\Gamma_{12}\right)\left(\rho_{S}-\left\langle e e\left|\rho_{T}\right| e e\right\rangle\right) .
\end{aligned}
$$

Here $H_{T}$ denotes the non-Hermitian Hamiltonian, corresponding to the interaction picture, which can be represented in the triplet part of the basis as follows:

$$
H_{T}=\left[\begin{array}{ccc}
-i \Gamma & \sqrt{2} E & 0 \\
\sqrt{2} E & \Omega-\frac{i}{2}\left(\Gamma+\Gamma^{(12)}\right) & \sqrt{2} E \\
0 & \sqrt{2} E & 0
\end{array}\right] .
$$

From Eq. (6) it is clearly seen that if $\Gamma=\Gamma_{12}$, the population of the antisymmetric state will remain constant, i.e., equa- tions of motion for $\rho_{T}$ and $\rho_{S}$ will decouple. In this case, there are two independent steady-state solutions. Otherwise there will be only one solution.

It is evident from Eq. (7) that in the absence of the classical driving field, all states except $|g g\rangle$ are damped, so that the steady-state entanglement at $E=0$ is impossible, and the system evolves towards the unentangled ground state $|g g\rangle$.

Because we are interested in the robust entanglement, let us consider the steady-state solutions of the master equation (6) for $\rho_{T}$. Consider first the Lamb-Dicke limit of short interatomic separation. Then, it follows from the definition of the decay rate (3) that

$$
\Gamma^{(12)} \approx \Gamma
$$

In this case, assuming that the atoms are initially prepared in their ground states, the steady-state density matrix will be determined in the triplet sector as follows:

$$
\rho_{T}=\frac{1}{N}\left[\begin{array}{ccc}
64 E^{4} & -16 i E^{3} \sqrt{2} & 8 E^{2}(2 i \Omega-1) \\
16 i E^{3} \sqrt{2} & 8 E^{2}\left(1+8 E^{2}\right) & -2 E \sqrt{2}\left(2 \Omega+i+8 i E^{2}\right) \\
-8 E^{2}(2 i \Omega+1) & -2 E \sqrt{2}\left(2 \Omega-i-8 i E^{2}\right) & 4\left(\Omega^{2}+2 E^{2}+16 E^{4}\right)+1
\end{array}\right] .
$$

Here $N$ is the normalization factor and $\Omega$ and $E$ are replaced by the dimensionless parameters $\Omega / \Gamma$ and $E / \Gamma$, respectively.

To determine the settings, leading to the maximum possible amount of entanglement in the system under consideration, we choose $\Omega=\tau E^{2}$, where $\tau$ is a dimensionless constant to be determined upon the maximization of concurrence. This factor in the Lamb-Dicke limit can be represented as follows:

$$
\tau=\frac{3}{4 \pi \alpha}\left[(k r)^{3} Q \bar{n} V\right]^{-1},
$$

where $\alpha=1 / 137$ is the fine-structure constant, $Q$ denotes atomic quality factor $\left(Q=\omega_{0} T\right.$, and $T$ is the lifetime of the excited atomic state), $\bar{n}$ is the mean number of photons per unit volume in classical driving field, and $V$ denotes the volume of interaction between atom and field, so that $\bar{n} V$ gives the mean number of photons interacting with atom during the time $T$.

The concurrence (measure of entanglement in the case of two-qubit system) is defined as follows [11]:

$$
C=\max \left(\lambda_{1}-\lambda_{2}-\lambda_{3}-\lambda_{4}, 0\right),
$$

where $\lambda$ denotes the spectrum of matrix $R=(\sqrt{\rho} \bar{\rho} \sqrt{\rho})^{1 / 2}$ and $\bar{\rho}$ denotes the complex conjugation of Eq. (8) in the so-called "magic basis" [11]. The maximum entangled state provides $C=1$, while the unentangled states give $C=0$.
One can see from Eq. (4) that at fixed $\tau$ and in the LambDicke limit $\vec{k}_{0} \cdot \vec{r} \ll 1$, both dimensionless parameters $\Omega / \Gamma, E / \Gamma \gg 1$. In this case, the density matrix (8) takes the form

$$
\rho_{T} \approx \frac{1}{\tau^{2}+48}\left(\begin{array}{ccc}
16 & 0 & 4 i \tau \\
0 & 16 & 0 \\
-4 i \tau & 0 & 16+\tau^{2}
\end{array}\right)
$$

To our surprise, the concurrence (10) in this limit turns out to be rational function of $\tau$,

$$
C(\tau)=\frac{8 \tau-16}{\tau^{2}+48}, \quad \tau \geqslant 2,
$$

extended by zero at $\tau \leqslant 2$. Thus entanglement is impossible if $\tau \leqslant 2$. The maximum value of the concurrence

$$
C_{\max }=\frac{2}{\sqrt{13}+1} \approx 0.43
$$

is attained at

$$
\tau_{\max }=2+2 \sqrt{13} \approx 9.21 .
$$

The corresponding amount of entanglement [11] is

$$
\mathcal{E}_{\max }=H\left(\frac{1-\sqrt{1-C_{\text {max }}^{2}}}{2}\right) \approx 0.285 \text { ebit. }
$$

Taking into account the form of the dimensionless parameter $\tau$ given by Eq. (9), we can examine the dimensionless inter- 


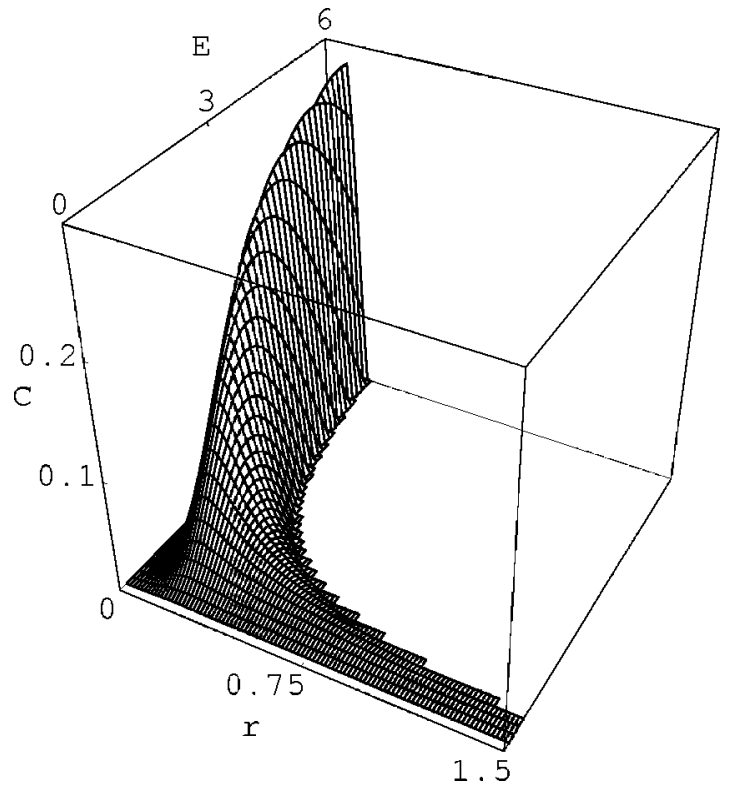

FIG. 1. Numerical dependence of concurrence on the interatomic distance and classical driving field. The dimensionless quantities $r / \lambda$ and $E / \Gamma$ are used here. $\lambda$ is the wavelength corresponding to atomic transition.

atomic distance $\vec{k}_{0} \cdot \vec{r}$, corresponding to the maximum entanglement provided by $\tau_{\max }=9.21$, as a function of the number of photons $\bar{n} V$, which should obey the condition $\bar{n} V \gg 1$ in the case of classical driving field. It is seen that in the case of a mean number of photons $\bar{n} V \sim 10$, the interatomic distance should be of the order of $10^{-2} \lambda$ (where $\lambda$ is the wavelength) to achieve the maximum possible amount of entanglement. An increase of the mean number of photons in the driving field, considered as a coherent state $|\alpha\rangle$ with $|\alpha|^{2} \gg 1$, decreases the interatomic distance, which is required to have a maximum amount of entanglement.

So far we have discussed the Lamb-Dicke limit. The results of numerical calculations beyond the Lamb-Dicke limit for different values of the classical driving field are shown in Fig. 1. Both cooperations, the dipole coupling and collective decay, are oscillating functions of distance [Eqs. (3) and (4)], and even when one of them becomes zero, the other can still give rise to entanglement (see Fig. 2). The deviation from the Lamb-Dicke limit decreases the cooperation effects, thus decreases steady-state entanglement.

Summarizing, we have examined the system of two identical two-level atoms, interacting with each other by means of vacuum induced dipole forces and collective decay. The dissipation of energy in the system is provided by the spontaneous decay of the excited atomic states. The compensation of losses is provided by a classical driving field.

It is shown that in the absence of the classical driving field, the system evolves towards an unentangled state (both atomic dipoles are in the ground state). The presence of the classical driving field stabilizes the entanglement.

In the Lamb-Dicke limit of a pointlike system, we obtained an exact solution for the steady-state density matrix, that manifests a high amount of entanglement (the concur-

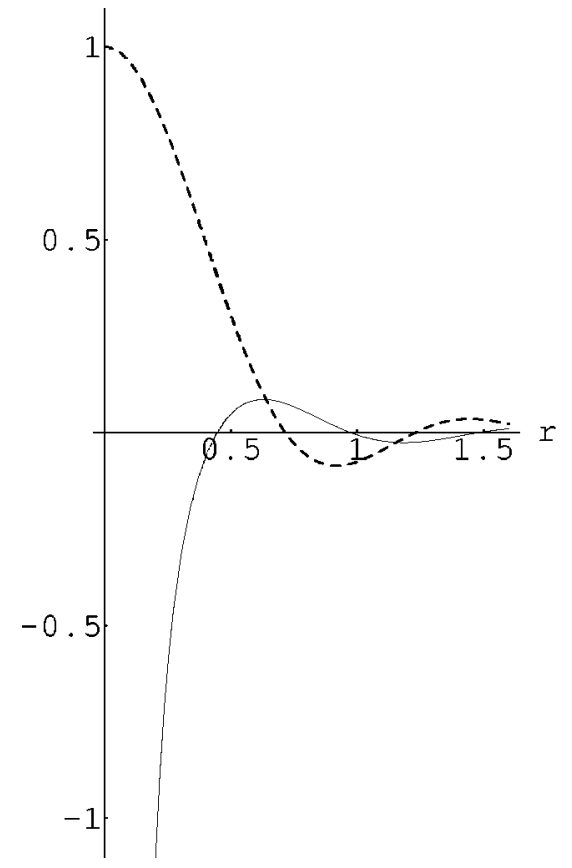

FIG. 2. The dipole interaction constant $\Omega$ [Eq. (4)] (dashed curve) and collective decay rate $\Gamma_{12}$ [Eq. (3)] (solid curve) as a function of interatomic separation $r$. Here $r$ is given in terms of wavelength corresponding to atomic transition.

rence $C_{\max }=0.43$ and the entanglement $\mathcal{E}_{\max }=0.285$ ebit $)$. This amount is much higher than in a number of recent proposals. In particular, it is higher than that in the case when the squeezed vacuum is used for stabilization of entanglement instead of the classical driving field [3].

Outside the Lamb-Dicke limit, i.e., when $\Gamma_{12}<\Gamma$, both the triplet and the singlet sectors of the density matrix (5) are populated, and this leads to a decrease in the amount of entanglement.

In free space small, interatomic distances are required for strong atomic cooperation. However, atoms can exhibit collective effects in cavities, or in the vicinity of dielectric bodies [12] even when they are spatially well separated. The prescribed scheme of steady-state entanglement generation can as well be applied to these cases.

In the above consideration, we always assumed that atoms are identical. It seems interesting to extend our consideration to the case of nonidentical atoms. In view of the result of Ref. [3], we can expect that this may lead to a significant increase of entanglement.

We also restricted our consideration to the case of polarization of the classical driving field parallel to the interatomic axis. The alternative choice of the polarization perpendicular to the interatomic axis can lead to a strong change of picture as well. First of all, the change of polarization changes the form of the coupling constant (4). Then, it causes the consideration of the different values of the classical driving field in the atomic locations.

The detailed analysis of the above-mentioned two extensions of the model deserves special consideration. 
[1] G. Raithel, C. Wagner, H. Walther, L. M. Narducci, and M. O. Scully, in Advances in Atomic, Molecular, and Optical Physics, edited by P. Berman (Academic Press, New York, 1994); J. M. Raymond, M. Brune, and S. Haroche, Rev. Mod. Phys. 73, 565 (2001); G. Rempe, Ann. Phys. 9, 843 (2000); H. Walther, in Quantum Communication and Information Technologies, edited by A. S. Shumovsky and V. I. Rupasov (Kluwer, Dordrecht, 2003).

[2] C. Monroe, D. M. Meekhof, B. E. King, and D. J. Wineland, Science 272, 1131 (1996); C. J. Miyatt, B. E. King, Q. A. Turchette, C. A. Sackett, D. Kielpinski, W. M. Itano, C. Monroe, and D. J. Wineland, Nature (London) 403, 269 (2000); D. J. Wineland et al., Philos. Trans. R. Soc. London, Ser. A 361, 1349 (2003); B. Julsgarland, A. Kozhekin, and E. S. Polzik, Nature (London) 413, 400 (2001); E. S. Polzik, ibid. 428, 129 (2004).

[3] R. Tanaś and Z. Ficek, J. Opt. B: Quantum Semiclassical Opt.
6, S610 (2004); quant-ph/0309195.

[4] S. G. Clark and A. S. Parkins, Phys. Rev. Lett. 90, 047905 (2003).

[5] B. Kraus and J. I. Cirac, Phys. Rev. Lett. 92, 013602 (2004).

[6] S. Nicolosi, A. Napoli, A. Messina, and F. Petruccione, Phys. Rev. A 70, 022511 (2004).

[7] J. B. Xu and S. B. Li, quant-ph/0401010.

[8] M. A. Can, Ö. Çakir, A. A. Klyachko, and A. S. Shumovsky, Phys. Rev. A 68, 022305 (2003).

[9] A. Biswas and G. S. Agarwal, J. Mod. Opt. 51, 1627 (2004). [10] R. H. Lehmberg, Phys. Rev. A 2, 889 (1970).

[11] Concerning concurrence as the measure of entanglement in the two-qubit systems, see S. Hill and W. K. Wootters, Phys. Rev. Lett. 78, 5022 (1997).

[12] H. T. Dung, S. Scheel, D. G. Welsch, and L. Knöll, J. Opt. B: Quantum Semiclassical Opt. 4, 169 (2002). 